\title{
Efeitos do propofol na resposta contrátil do miocárdio à dopamina e dobutamina: estudo experimental em corações isolados de ratos
}

\author{
José Carlos Dorsa PONTES ${ }^{\star}$, Carlos Geraldo Sobral MEDEIROS ${ }^{\star \star}$, Otoni M. GOMES ${ }^{\star *}$
}

RBCCV $44205-323$

\begin{abstract}
Pontes J CD, Medeiros C GS, Gomes O M-Efeitos do propofol na resposta contrátil do miocárdio à dopamina e dobutamina: estudo experimental em corações isolados de ratos. Rev Bras Cir Cardiovasc 1996; $11(4): 292-8$.
\end{abstract}

RESUMO:Objetivo: Estudo experimental das ações farmacodinâmicas do propofol e sua interação com a dopamina e dobutamina em coraçōes isolados de ratos.

Método: Foram estudadas as variaçōes da contratilidade miocárdica (dT/dt), em 30 corações isolados de ratos. Em todos os animais, após anestesia por inalaçāo de éter, os coraçōes foram excisados e perfundidos em sistema de Langendorff com solução de Krebs - Hensleit enriquecida com $95 \% \mathrm{O}_{2}$ e $5 \% \mathrm{CO}_{2}$ ' (pressão de $90 \mathrm{~cm}$ de $\mathrm{H}_{2} \mathrm{O}$, temperatura constante de $37,0^{\circ} \mathrm{C} \pm 0,5^{\circ} \mathrm{C}$ ). Foram estudados 30 animais divididos em: Grupo I (controle) - 10 coraçōes perfundidos durante 11 minutos com soluçāo de Krebs - Hensleit; Grupo II (dopamina-propofol-dopamina) - 10 coraçōes onde foram administrados dopamina ( $50 \mathrm{mcg} / \mathrm{ml})$ e analisados os resultados nos $1^{\circ}, 3^{\circ}$ e $5^{\circ}$ minutos e, posteriormente, propofol, $(25 \mathrm{mcg} / \mathrm{ml})$ infundindo-se 1 minuto após, dopamina $\left(50 \mathrm{mcg} / \mathrm{ml}\right.$ ) e analisando-se os $1^{\circ}, 3^{\circ}$ e $5^{\circ}$ minutos. Grupo III (dobutamina-propofoldobutamina) - diferiu do Grupo II pela substituição da dopamina por dobutamina $(50 \mathrm{mcg} / \mathrm{ml})$.

Resultados: No Grupo I observou-se que a dT/dt variou de 39,57 $\pm 3,97$ (g.seg ${ }^{-1}$ ) a 39,37 $\pm 3,44$ (g.seg ${ }^{-1}$ ) $(p>0,05)$ no periodo estudado. NoGrupoll observou-se que, após a administração de propofol e dopamina, a dT/dt em $\left(\right.$ g.seg $\left.^{-1}\right)$ apresentou queda de $17,61 \%(p<0,05)$ no $1^{2}$ minuto; $8,37 \%(p<0,05)$ no $3^{\circ}$ minuto e $10,84 \%(p<0,05)$ no $5^{\circ}$ minuto, comparado à injeção isolada da dopamina. No Grupo III observou-se que, após a administração de propofol e dobutamina a dT/dt em $\left(\mathrm{g}_{\mathrm{seg}} \mathrm{se}^{-1}\right)$ sofreu acréscimo de $6,16 \%(p>0,05)$ no $1^{\circ}$ minuto; $3,62 \%(p>0,05)$ no $3^{\circ}$ minuto e $3,08 \%(p>0,05)$ no $5^{\circ}$ minuto, comparado à injeçāo isolada da dobutamina.

Conclusão: O propofol $(25 \mathrm{mcg} / \mathrm{ml})$ não alterou a resposta contrátil do miocárdio à dobutamina $(50 \mathrm{mcg} /$ $\mathrm{ml})$; no entanto, inibiu a resposta esperada pela ação da dopamina $(50 \mathrm{mcg} / \mathrm{ml})$ na contratilidade miocárdica.

DESCRITORES: Contraçāo miocárdica, efeitos de drogas. Propofol, farmacologia. Dopamina, farmacologia. Dobutamina, farmacologia. Ratos, estudos experimentais.

Trabalho realizado no Centro de Pesquisas Experimentais do Serviço do Coração H.S.F.A. Belo Horizonte, MG, Brasil e no Laboratório de Cirurgia Cardiovascular Experimental da Disciplina de Cirurgia Cardiovascular do Departamento de Clínica Cirúrgica da Universidade Federal de Mato Grosso do Sul, MS, Brasil.

Apresentado ao $23^{\circ}$ Congresso Nacional de Cirurgia Cardiaca. Recife, PE, 20 a 23 de março, 1996.

* Do departamento de Clínica Cirúrgica da Universidade de Mato Grosso do Sul.

** Do Serviço do Coração H.S.F.A. - Belo Horizonte.

Endereço para correspondência: José Carlos Dorsa V. Pontes. Rua Sergipe 73, Vila Célia. Campo Grande - MS, Brasil. CEP: $79020-161$. 
Pontes J CD, Medeiros C G S, Gomes O M - Efeitos do propofol na resposta contrátil do miocárdio à dopamina e dobutamina: estudo experimental em coraçōes isolados de ratos.

Rev Bras Cir Cardiovasc 1996; 11 (4):292-8 .

\section{INTRODUÇÃO}

Com o desenvolvimento da técnica cirúrgica e o maior conhecimento da anatomia e da fisiopatologia, a cirurgia cardíaca, como proposta terapêutica, passou a abordar e tratar lesões cardiacas cada vez mais complexas.

Para que se atingissem resultados progressivamente melhores, houve necessidade de se otimizar a técnica operatória, o procedimento anestésico e o controle pós-operatório.

No tocante à anestesia, várias substâncias vêm sendo usadas. Observa-se, contudo, que essas drogas podem determinar graus variados de depressão miocárdica. O propofol é um anestésico não barbitúrico, derivado fenólico (2,6 - disopropil Fenol), altamente lipofílico e insolúvel em água. Tem conquistado espaço dentro do arsenal de drogas usadas em anestesia para cirurgia cardíaca. Possui meia vida curta, rápida distribuição e eliminação com elevada depuração plasmática (1-4)

Vários autores ${ }^{(5-8)}$ fazem referências ao propofol como determinante de diminuição da contratilidade miocárdica podendo desencadear desequilibrio hemodinâmico em pacientes com afecções cardiovasculares graves.

COATES et al. (9), CLAEYS et al. (10), GAUSS et al. (11) relataram, no homem, após injeções únicas do propofol, na dose de $2,0 \mathrm{mg} / \mathrm{kg}$, importantes alterações hemodinâmicas com diminuição do inotropismo do miocárdio, da resistência vascular sistêmica, das pré e pós cargas, além de hipotensões arteriais de leve a moderada intensidade.

BRUSSEL et al. (5), WOÜTERS et al. (12), PUTTICK et al (13), GOODCHILD \& SERRAO (14) demonstraram que o efeito depressor do miocárdio com uso do propofol é dose dependente.

NASCIMENTO et al. (15), em estudo experimental em cães, obtiveram maior estabilidade hemodinâmica com infusão contínua e baixas doses desse medicamento.

MEDEIROS (16), em corações isolados de ratos, em preparação de Langerdorff utilizando propofol $25 \mathrm{mcg} / \mathrm{ml}$ e $50 \mathrm{mcg} / \mathrm{ml}$, concluiu que tal droga determinou depressão na contratilidade miocárdica em ambas situações.

O suporte inotrópico tem sido freqüente em cirurgia cardiaca, em função do estresse das intervençōes, da gravidade das lesões abordadas, da reserva miocárdica e até mesmo em função da depressão da contratilidade miocárdica determinada por outras drogas utilizadas na anestesia.

COETZEE et al. (17), BRUSSEL et al. (5) e RIOU et al. (18) demonstraram diminuição da contratilidade miocárdica induzida pelo propofol em experimentação animal fazendo alusão ao fato do propofol diminuir o influxo de cálcio no miócito.

DI FLÓRIO (19) levantou a hipótese do propofol bloquear o receptor dopaminérgico (D2). Essa hipótese tem sido suportada por relatos de crises oculogíricas $(2,20)$ e elevados níveis de prolactina após anestesia com propofol (21).

Uma vez que os agentes inotrópicos dopamina e dobutamina agem aumentando o influxo de cálcio no miócito e considerando que a ação depressora da contratilidade miocárdica determinada pelo propofol ainda é motivo de indagações a cerca do bloqueio de receptores e diminuição de influxo de cálcio na célula miocárdica, aventa-se a possibilidade do propofol modificar a resposta cardiaca aos inotrópicos.

Face ao exposto e considerando a escassez de publicaçōes a respeito de interações de inotrópicos e propofol, a presente investigação se propõe a analisar a resposta contrátil do miocárdio em corações isolados de ratos tratados com dopamina e dobutamina, concomitantemente à ação desta droga.

\section{MATERIAL E MÉTODOS}

Foram estudados 30 ratos albinos, raça Wistar, provenientes do Biotério do Serviço do Coração do Hospital São Francisco de Assis/C.M.C./S.S.V.P. distribuídos nos seguintes grupos:

\section{Grupo I - (Controle)}

Dez corações, mantidos em perfusão com solução padrão (Krebs-Hensleit) durante 11 minutos a partir da obtenção dos registros de controle, ou seja 15 minutos após acoplamento do coração isolado ao sistema de perfusão, sendo os parâmetros registrados no $1^{\circ}, 3^{\circ}, 5^{\circ}$ e $11^{\circ}$ minutos.

\section{Grupo II - (Dopamina-Propofol-Dopamina)}

Dez corações submetidos a perfusão com solução padrão durante 15 minutos. Após obtenção dos registros controles, foram infundidos 50 microgramas de dopamina no circuito de perfusão coronariana. Analisaram-se os $1^{\circ}, 3^{\circ}$ e $5^{\circ}$ minutos. Procedeu-se à administração de 25 microgramas de propofol. Um minuto após, foram administrados $50 \mathrm{mi}-$ crogramas de dopamina, observando-se os $1^{\circ}, 3^{\circ} \mathrm{e}$ $5^{2}$ minutos seguintes.

\section{Grupo III - (Dobutamina-Propofol-Dobutamina)}

Dez corações submetidos à perfusão com solução padrão durante 15 minutos. Após obtenção dos registros controles, foram infundidos 50 microgramas de dobutamina no circuito de perfusão coronariana. Analisaram-se os $1^{\circ}, 3^{\circ}$ e $5^{\circ}$ minutos. Pro- 
Pontes J CD, Medeiros C G S, Gomes OM - Efeitos do propofol na resposta contrátil do miocárdio à dopamina e dobutamina: estudo experimental em coraçōes isolados de ratos. Rev Bras Cir Cardiovasc 1996; 11 (4):292-8 .

cedeu-se à administração de 25 microgramas de propofol. Um minuto após, administraram-se $50 \mathrm{mi}-$ crogramas de dobutamina, observando-se os $1^{\circ}, 3^{\circ}$ e $5^{2}$ minutos seguintes.

Os animais foram anestesiados por inalação de éter sulfúrico em campânula fechada. Realizou-se toracotomia ampla, com ressecção do plastrão esternocostal para exposição do coração e grandes vasos. Após administração de 500 unidades de heparina sódica, por punção direta da veia cava inferior, a aorta ascendente foi canulada. $O$ ventrículo esquerdo foi drenado através de cânula multifenestrada introduzida pelo átrio esquerdo e exteriorizada pelo seu ápice, evitando-se lesão de artérias coronárias principais.

Os corações suspensos pelas cânulas de perfusão aórtica, após terem sido excisados, foram, então, unidos ao sistema de perfusão coronariana.

Empregou-se o método de LANGENDORFF (22), com perfusato de solução de KREBS \& HENSLEIT (23), gaseificada com $95 \%$ de $\mathrm{O}_{2}$ e $5 \% \mathrm{CO}_{2}$ obtendo-se $\mathrm{pH}$ de $7,35 \pm 0,05$ (solução padrão). A pressão de perfusão foi mantida em $90 \mathrm{~cm}$ de $\mathrm{H}_{2} \mathrm{O}$ e a temperatura em $37^{\circ} \mathrm{C} \pm 05^{\circ} \mathrm{C}$, utilizando-se unidade de aquecimento contendo módulo descartável (Flumen Produtos Médicos Ltda.).

Após perfusão durante 15 minutos para estabilização da atividade cardiaca, os coraçōes foram acoplados ao sistema de registro de contratilidade miocárdica.

Para estudo da contratilidade miocárdica, empregou-se um transdutor de força modelo Grass (force displacement tranducer model FT 03 - Grass Instrument Company), acoplado ao ápice do coração por sistema de roldana com microrrolamento, aperfeiçoamento técnico ${ }^{(24)}$, modificado por PITCHON (25).

Os registros de Tensão $(T)$, Freqüência Cardiaca (bpm) e Temperatura foram processados por biomotor (Bese - Bioengenharia $\mathrm{DH} 073$ ) e impresso, para análise e estudo comparativo.

Foram estudadas as variações das Derivadas Tensão/Tempo (dT/dt) referidas em gramas segundo ${ }^{-1}$.

\section{RESULTADOS}

Os seguintes resultados foram obtidos nos diferentes grupos estudados:

A Tabela 1 demonstra as variações da Dt/dt (g.seg ${ }^{-1}$ ) do Grupo I (controle). Houve queda de $2,52 \%$ no $5^{\circ}$ minuto e $0,51 \%$ no $11^{\circ}$ minuto, não sendo estas variações estatisticamente significativas.

A Tabela 2 demonstra as variações da $\mathrm{dT} / \mathrm{dt}$ (g. seg $^{-1}$ ) do Grupo II (dopamina-propofol-dopamina) onde foram comparados os $1^{\circ}, 3^{\circ}$ e $5^{\circ}$ minutos do uso isolado da dopamina com os $1^{\circ}, 3^{\circ}$ e $5^{\circ}$ minutos do uso da dopamina pós propofol. Verificou-se queda da dT/dt em (g.seg ${ }^{-1}$ ): de $56,83 \pm 9,73$ para 49,99 $\pm 8,49$ $(\mathrm{p}<0,05)$ no $1^{9}$ minuto; de $51,83 \pm 8,44$ para $48,58 \pm$ $8,37(\mathrm{p}<0,05)$ no $3^{\circ}$ minuto e de $40,66 \pm 8,54$ para $36,49 \pm 8,90(p<0,05)$ no $5^{\circ}$ minuto.

A Tabela 3 demonstra as variações da $\mathrm{dT} / \mathrm{dt}$ (g.seg ${ }^{-1}$ ) do Grupo III (dobutamina-propofoldobutamina) onde foram comparados os $1^{\circ}, 3^{\circ}$ e $5^{\circ}$ minutos do uso isolado da dobutamina com os $1^{\circ}, 3^{\circ}$ e $5^{\circ}$ minutos do uso da dobutamina pós propofol. Verificou-se que houve acréscimo na dT/dt em (g.seg ${ }^{-1}$ ): de $38,49 \pm 6,17$ para $56,00 \pm 11,79(p>0,05)$ no $1^{\circ}$

TABELA 1

GRUPO I (controle): VARIAÇĀO DA dT/dt $\left(\right.$ g. $\left.^{\mathrm{seg}^{-1}}{ }^{-1}\right)$

\begin{tabular}{|c|c|c|c|c|c|}
\hline $\begin{array}{c}\text { OBS } \\
N^{2}\end{array}$ & CONTROLE & 1 & $\begin{array}{c}\text { PERIODO } \\
3\end{array}$ & 5 & $11 \mathrm{MIN}$ \\
\hline 1 & 42,50 & 42,50 & 42,50 & 41,25 & 41,25 \\
\hline 2 & 41,25 & 41,25 & 41,25 & 40,00 & 40,00 \\
\hline 3 & 42,50 & 42,50 & 42,50 & 40.00 & 40,00 \\
\hline 4 & 40,00 & 40,00 & 40,00 & 40,00 & 40,00 \\
\hline 5 & 42,00 & 42,00 & 42,00 & 41,25 & 42,50 \\
\hline 7 & 37,50 & 37,50 & 37,50 & 37,50 & 40,00 \\
\hline 8 & 40,00 & 40,00 & 40,00 & 40,00 & 38,75 \\
\hline 9 & 37,50 & 37,50 & 37,50 & 37,50 & 40,00 \\
\hline 10 & 32,50 & 32,50 & 32,50 & 30,00 & 30,00 \\
\hline MÉDIA & 39,57 & 39,57 & 39,57 & 39,00 & 39,37 \\
\hline D.P. & 3,07 & 3,07 & 3,07 & 3,52 & 3,44 \\
\hline$p$ & 0,12 & 0,12 & 0,12 & 0,12 & 0,19 \\
\hline
\end{tabular}


Pontes J CD, Medeiros C G S, Gomes O M - Efeitos do propofol na resposta contrátil do miocárdio à dopamina e dobutamina: estudo experimental em coraçōes isolados de ratos. Rev Bras Cir Cardiovasc 1996; 11 (4):292-8.

TABELA 2

GRUPO II (dopamina-propofol-dopamina): VARIAÇÃO DA dT/dt - $\left(g . s e g .^{-1}\right)$

\begin{tabular}{ccccccccc}
\hline $\begin{array}{c}\text { OBS } \\
\mathbf{N}^{\mathbf{S}}\end{array}$ & CONTROLE & $\mathbf{1}$ & $\begin{array}{c}\mathbf{3} \\
\text { DOPAMINA }\end{array}$ & $\mathbf{5}$ & $\begin{array}{c}\text { PERIODO } \\
\mathbf{1} \\
\text { PROPOFOL }\end{array}$ & $\mathbf{1}$ & $\begin{array}{c}\mathbf{3} \\
\text { DOPAMINA }\end{array}$ \\
\hline 1 & 33,33 & 55,00 & 55,00 & 45,00 & 33,33 & 36,66 & 42,50 & 26,66 \\
2 & 32,5 & 51,66 & 43,33 & 33,33 & 30,00 & 51,66 & 43,33 & 30,00 \\
3 & 30,00 & 40,00 & 40,00 & 30,00 & 25,00 & 40,00 & 36,66 & 26,66 \\
4 & 25,00 & 43,33 & 40,00 & 33,33 & 22,50 & 40,00 & 36,66 & 30,00 \\
5 & 37,50 & 56,66 & 56,66 & 50,00 & 32,50 & 60,00 & 55,00 & 43,33 \\
6 & 50,00 & 70,00 & 50,00 & 30,00 & 28,33 & 60,00 & 55,00 & 33,33 \\
7 & 53,33 & 60,00 & 53,33 & 53,33 & 32,50 & 50,00 & 50,00 & 50,00 \\
8 & 40,00 & 60,00 & 65,00 & 40,00 & 33,33 & 55,00 & 60,00 & 50,00 \\
9 & 40,00 & 65,00 & 55,00 & 45,00 & 17,50 & 50,00 & 50,00 & 35,00 \\
10 & 46,66 & 66,66 & 60,00 & 46,66 & 33,33 & 56,66 & 56,66 & 40,00 \\
\hline MÉDIA & 38,83 & 56,83 & 51,83 & 40,66 & 28,83 & 49,99 & 48,58 \\
D.P. & 9,07 & 9,73 & 8,44 & 8,54 & 5,50 & 8,49 & 8,37 \\
P & & 0,0065 & 0,02 & 0,05 & 0,0007 & 0,0065 & 0,02 \\
\hline
\end{tabular}

minuto; de $53,66 \pm 9,98$ para $55,00 \pm 11,36(p>0,05)$ no $3^{\circ}$ minuto e de $52,08 \pm 8,72$ para $53,25 \pm 12,75$ ( $p>0,05)$ no $5^{2}$ minuto.

A Tabela 4 e o Gráfico 1 apresentam os resultados das variações porcentuais médias da $\mathrm{dT} / \mathrm{dt}$ de cada grupo estudado, verificando-se que, no grupo controle, não ocorreram alteraçōes estatisticamente significativas nos periodos estudados.

As variações porcentuais médias da $\mathrm{dT} / \mathrm{dt}$ no uso isolado da dopamina comparada ao uso da dopamina após administração do propofol sofreram queda estatisticamente significativa em todos os momentos estudados.

As variações médias da $\mathrm{dT} / \mathrm{dt}$ no uso isolado da dobutamina comparada com sua injeção após administração do propofol não sofreram variações estatisticamente significativa.

\section{GRÁFICO 1}

VARIAÇĀO PORCENTUAL MÉDIA DA dT/dT NOS GRUPOS ESTUDADOS

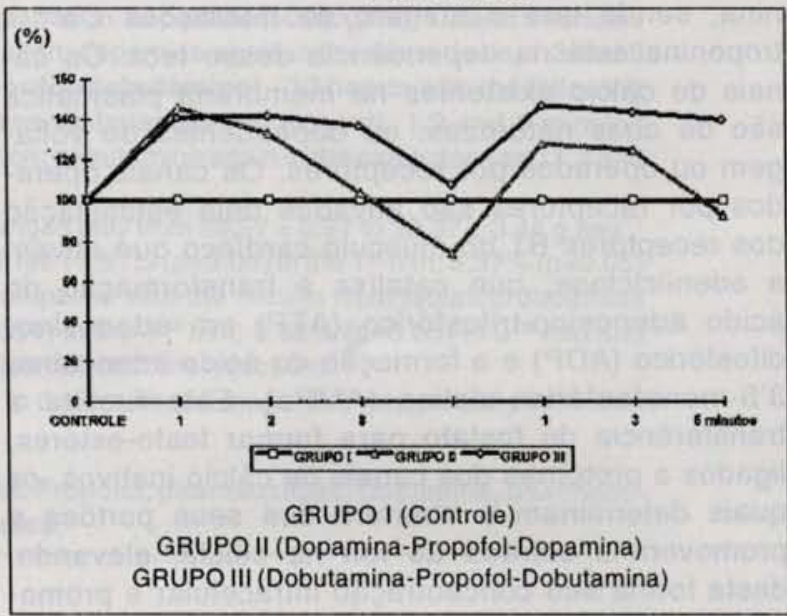

TABELA 3

GRUPO III (DOBUTAMINA-PROPOFOL-DOBUTAMINA): VARIAÇÃO DAdT/dt-(g.seg-1)

\begin{tabular}{|c|c|c|c|c|c|c|c|c|}
\hline $\begin{array}{l}\text { OBS } \\
N^{2}\end{array}$ & controle & 1 & $\stackrel{3}{\text { DOBUTAMINA }}$ & 5 & $\begin{array}{c}\text { PERIOODO } \\
1 \\
\text { PROPOFOL }\end{array}$ & 1 & $\stackrel{3}{\text { DOBUTAMINA }}$ & $5 \mathrm{~min}$ \\
\hline 1 & 45,00 & 65,00 & 65,00 & 55,00 & 40,00 & 60,00 & 50,00 & 45,00 \\
\hline 2 & 33,33 & 57,50 & 55,00 & 45,00 & 35,00 & 45,00 & 40,00 & 37.5 \\
\hline 3 & 36,66 & 57,50 & 57,50 & 57,50 & 55,00 & 57,50 & 57,50 & 55,00 \\
\hline 4 & 40,00 & 56,66 & 53,33 & 53,33 & 53,33 & 70,00 & 70,00 & 70,00 \\
\hline 5 & 43,33 & 70,00 & 70,00 & 70,00 & 53,33 & 75,00 & 75,00 & 75,00 \\
\hline 6 & 36,66 & 40,00 & 52,50 & 52,50 & 40,00 & 60,00 & 55,00 & 55,00 \\
\hline 7 & 26,60 & 40,00 & 40,00 & 37,50 & 33,33 & 40,00 & 45,00 & 40,00 \\
\hline 8 & 43,33 & 50,00 & 50,00 & 50,00 & 33,33 & 45,00 & 50,00 & 50,00 \\
\hline 9 & 36,66 & 55,00 & 55,00 & 55,00 & 46,66 & 62,50 & 62,50 & 62,50 \\
\hline 10 & 38,33 & 45,00 & 45,00 & 45,00 & 28,33 & 45,00 & 45,00 & 42,50 \\
\hline MÉDIA & 38,49 & 53,66 & 54,33 & 52,08 & 41,33 & 56,00 & 55,00 & 53,25 \\
\hline D.P. & 6,17 & 9,98 & 8,72 & 8,73 & 9,66 & 11,79 & 11,36 & 12,75 \\
\hline$p$ & & 0,22 & 0,41 & 0,32 & 0,0003 & 0,22 & 0,41 & 0,32 \\
\hline
\end{tabular}


Pontes J CD, Medeiros C G S, Gomes O M - Efeitos do propofol na resposta contrátil do miocárdio à dopamina e dobutamina: estudo experimental em coraçōes isolados de ratos. Rev Bras Cir Cardiovasc 1996; 11 (4):292-8 .

TABELA 4

VARIAÇŌES PORCENTUAIS MÉDIAS DA dT/dT NOS GRUPOS ESTUDADOS

\begin{tabular}{|c|c|c|c|c|c|c|c|c|}
\hline GRU & CONTROLE & 1 & 3 & 5 & PERIOODO & 1 & 3 & $5 \mathrm{~min}$ \\
\hline 1 & 100 & $\begin{array}{c}100 \\
(p=0,1)\end{array}$ & $\begin{array}{c}100 \\
(p=0,1)\end{array}$ & $\begin{array}{c}100 \\
(p=0,1)\end{array}$ & $\begin{array}{c}100 \\
(p=0,1)\end{array}$ & $\begin{array}{c}100 \\
(p=0,1)\end{array}$ & $\begin{array}{c}100 \\
(p=0,1)\end{array}$ & $\begin{array}{c}100 \\
(p=0,1)\end{array}$ \\
\hline II & 100 & $\begin{array}{c}146 \\
(p=0,006)\end{array}$ & $\begin{array}{c}133 \\
(p=0,02)\end{array}$ & $\begin{array}{c}104 \\
(p=0,05)\end{array}$ & $\begin{array}{c}74 \\
(p=0,0007)\end{array}$ & $\begin{array}{c}128 \\
(p=0,0006)\end{array}$ & $\begin{array}{c}125 \\
(p=0,02)\end{array}$ & $\begin{array}{c}93 \\
(p=0,05)\end{array}$ \\
\hline III & 100 & $\begin{array}{c}141 \\
(p=0,2)\end{array}$ & $\begin{array}{c}142 \\
(p=0,4)\end{array}$ & $\begin{array}{c}137 \\
(p=0,6)\end{array}$ & $\begin{array}{c}108 \\
(p=0,0003)\end{array}$ & $\begin{array}{c}147 \\
(p=0,2)\end{array}$ & $\begin{array}{c}144 \\
(p=0,4)\end{array}$ & $\begin{array}{c}140 \\
(p=0,6)\end{array}$ \\
\hline
\end{tabular}

Nivel de significância: $p<0,05$

Os valores de "p" nos grupos II e III referem-se às análises estatísticas das variaçōes ocorridas entre os momentos do uso isolado dos inotrópicos pareados aos momentos das interaçōes entre inotrópicos e propofol.

\section{COMENTÁRIOS}

Durante a contração do miócito e o acoplamento eletromecânico, a elevação do teor de cálcio livre no sarcômero promove a sua interação com a troponina, sendo que o número de interações $\mathrm{Ca}^{++}$. troponina está na dependência desse teor. Os canais de cálcio existentes na membrana plasmática são de duas naturezas: ou dependentes de voltagem ou operados por receptores. Os canais operados por receptores são ativados pela estimulação dos receptores B1 do músculo cardíaco que ativam a adenilciclase, que catalisa a transformação do ácido adenosino-trifosfórico (ATP) em adenosinodifosfórico (ADP) e a formação do ácido adenosino3'5-monofosfórico-cíclico (AMPc). Este facilita a transferência do fosfato para formar fosfo-estéres, ligados a proteinas dos canais de cálcio inativos, os quais determinam a abertura dos seus portões e promovem a entrada do íon na célula, elevando, desta forma sua concentração intracelular e promovendo aumento de força contrátil.

Dessa maneira excercem efeito inotópico atuando como angonistas beta-adrenérgicos:a dopamina (precursor metabólico imediato da adrenalina e noradrenalina) e a dobutamina (sintética, semelhante quimicamente à dopamina, possuindo um substituto aromático volumoso no grupo amino).

\section{ANÁLISE ESTATISSTICA}

Os resultados obtidos foram submetidos a estudo estatístico, aplicando-se o Teste T de Student para comparações pareadas (26), sendo estabelecido o nível de significância em 0,05 . Foi utilizado o software Excel 4.0, da Microsoft do Departamento de Computação e Estatística do Centro de Ciências Exatas e Tecnologia da FUFMS.

Os efeitos cardiovasculares do propofol foram estudados por diversos autores $(9,27,28)$, onde observaram decréscimo estatisticamente significativo de $30 \%$ da pressão sistólica, aproximadamente. Concluíram que ocorre decréscimo da pressão arterial em função da queda da resistência vascular sistêmica e não em função da queda do débito cardíaco.

Outros autores (29-31), analisando pacientes com boa função ventricular anestesiados com propofol e submetidos a revascularização miocárdica, observaram importante queda da pressão arterial, sem, contudo, ocorrência de mudanças significativas do índice cardíaco.

CLAEYS et al (10), estudando pacientes submetidos a cirurgias não cardíacas, não encontraram redução significativa do débito cardiaco, mas sim redução da resistência vascular sistêmica, assim como PATRICK et al. (29) e STEPHAN et al. (30).

LARSEN et al. (32) encontraram significante decréscimo do índice cardíaco, atribuindo a isso um efeito inotrópico negativo do propofol. Ficando, assim, conflitante a literatura, no que diz respeito ao efeito determinante da hipotensão arterial com uso do propofol.

No presente modelo experimental, onde a contratilidade pôde ser analisada de maneira direta, sem interferências de outros fatores como pré-carga e pós-carga, verificou-se que nos Grupos II e III, após administração de $25 \mathrm{mcg} / \mathrm{ml}$ de propofol, houve queda da contratilidade de $33,67 \% \quad(p<0,05)$ e $28,29 \%(p<0,05)$, respectivamente.

O propofol na dose de $25 \mathrm{mcg} / \mathrm{ml}$ determinou menor efeito da dopamina $(50 \mathrm{mcg} / \mathrm{ml})$ sobre a contratilidade miocárdica, do que durante uso isolado desse inotrópico. Houve queda estatisticamente significativa $(p<0,05)$ nos valores obtidos $d a d T / d t$ $17,61 \%, 8,37$ e $10,84 \%$, respectivamente nos $1^{\circ}, 3^{\circ}$ e $5^{\circ}$ minutos estudados após a interação das drogas. 
Pontes J C D, Medeiros C G S, Gomes O M-Efeitos do propofol na resposta contrátil do miocárdio à dopamina e dobutamina: estudo experimental em coraçōes isolados de ratos. Rev Bras Cir Cardiovasc 1996; 11 (4):292-8 .

Não ocorreram variações estatisticamente significativas da $\mathrm{dT} / \mathrm{dt}$ entre os momentos estudados com uso isolado da dobutamina comparados à associação de dobutamina e propofol, havendo, contudo, acréscimo de $6,16 \%$ ( $p>0,05), 3,62 \%$ ( $>>0,05 \%)$ e $3,08 \%(p>0,05)$, respectivamente nos $1^{\circ}, 3^{\circ}$ e $5^{\circ}$ minutos estudados com interação das drogas.

Do exposto, pode-se concluir que o propofol determinou depressão miocárdica e inibição da resposta à dopamina, nos corações estudados. Respeitadas as condições da presente investigação, parece mais recomendável a utilização da dobutamina, para reversão de efeitos depressores miocárdicos induzidos pelo uso do propofol para sedação em unidades coronarianas, C.T.I. ou durante operações cardiovasculares.

\section{RBCCV 44205-323}

Pontes J C D, Medeiros C G S, Gomes O M - Effects of the propofol in the myocardial contractile response to dopamine and dobutamine in isolated rat's heart Rev Bras Cir Cardiovasc 1996; 11 (4): 292-8 .

ABSTRACT: Purpose: Experimental investigation of the pharmacodynamic effects of propofol with and without simultaneous injections of dopamine or dobutamine in isolated hearts of rats.

Methods: In all animals under anaesthesia the hearts were removed and irrigated by a Krebs-Hensleit solution containing $\mathrm{O}_{2}(95 \%)$ and $\mathrm{CO}_{2}(5 \%)$, under constant pressure of $90 \mathrm{~cm} \mathrm{H}_{2} \mathrm{O}$ and at $37^{\circ} \mathrm{C}$. After stabilization of the heart activity during 15 minutes, the control values were obtained. The following groups were considered: Group I (control) - 10 hearts perfused only with the Krebs-Hensleit solution during 11 minutes after the control values registration. Group II (dopamine-propofol-dopamine) - 10 hearts with the following sequency of drug injections and results values registration: dopamine $(50 \mathrm{mcg} / \mathrm{ml}), 1.3$ and 5 minutes registration; propofol $(25 \mathrm{mcg} / \mathrm{ml}), 1$ minute period registration; dopamine again in the same dosis, 1.3 and 5 minutes values registration. Group III (dobutamine-propofol-dobutamine) - 10 hearts with the following sequency of drug injections and results values registration: dobutamine $(50 \mathrm{mcg} / \mathrm{ml}), 1.3$ and 5 minutes registration: propofol ( $25 \mathrm{mcg} / \mathrm{ml}), 1$ minute period registration; dobutamine again in the same dose and 1.3 and 5 minutes of registration.

Results: In Group I hearts, the dT/dt mean values changed only from $39.57 \pm 3.97$ to $39.37 \pm 3.44{\mathrm{~g} . \mathrm{seg}^{-1}}^{-1}$ ( $p>0.05)$. In Group II after propofol and dopamine the dT/dt fell $17.61 \%(p<0.05)$ in the $1^{\text {st }} \min ; 8.37 \%(p<0.05)$ in the $3^{\text {rd }} \mathrm{min}$ and $10.84 \%(p<0.05)$ in $5^{\text {th }} \mathrm{min}$, when compared with the results from isolated dopamine injections. In Group III the dT/dt increased 6.16\% $(p>0.050)$ in the $1^{\text {st }} \min ; 3.62 \%(p<0.05)$ in $3^{\text {rd }} \min$ and $3.08 \%(p>0.05)$ in the $5^{\text {th }} \mathrm{min}$, over the values from isolated dobutamine injections.

Conclusions: The myocardial contractile response to dopamine $\left(50 \mathrm{mcg}^{-\mathrm{ml}^{-1}}\right)$ was partially inhibited; by propofol $\left(25 \mathrm{mcg} / \mathrm{ml}^{-1}\right)$ action was not altered.

DESCRIPTORS: Mypcardial contraction, drug effects. Propofol, pharmacology. Dopamine, pharmacology. Dobutamine, pharmacology. Rats, experimental studies.

\section{REFERÊNCIAS BIBLIOGRÁFICAS}

1 Cockshotti I D, Briggs L P, Douglas E J, White M . Pharmacokinetics of propofol in female patients. $\mathrm{Br}$ $J$ Anaesth 1987; 59: 1103-10.

2 Rosengarten $\mathrm{H}$, Schweitzer J W, Friedhoff A J - Induction of oral dyskinesias in native rats by $D 1$ stimulation. Life Sci 1983; 33: 479.

3 Simmons P J, Cockshott I D, Douglas E J, Gordon E A, Hopkins $K$, Rowland $M$ - Disposition in male volunteers of a subanaesthetic intravenous dose of an oil in water emulsion of $14 \mathrm{C}$ - propofol. Xenabiotica 1988; 18: 429-40.

4 Twersky R S - The pharmacology of anesthetics used for ambulatory surgery. ASA Annual Refresher Courses Lectures 1993; 21: 159-75.
5 Brusel T, Theissen J L, Vigfusson $G$, Lukenheimer $P$, Van Aken $H$, Lawin P - Hemodynamic and cardiodynamic effects of propofol etomidate negative inotropic properties of propofol. Anesth Analg 1989; 69: $35-40$.

6 Pagel D S \& Waltier D C - Negative inotropic of propofol as evaluated by the regional preload recruitable stroke work relationship in chronically instrumented dogs. Anesthesiology 1993; 78: 100-8.

7 Puttick R M \& Tenar D A - Effects of propofol and anflurane on action potencials membrane currents and contraction of guinea-pig isolated ventricular myocytes. Br J Pharmacol 1992; 107: 559-65.

8 Shyr $\mathrm{M} \mathrm{H}$, Yang C A, Kuo T B, Pan W H, Tan P P, Chan $\mathrm{S} \mathrm{H}$ - Power spectral analysis of the electroencephalografic and hemodynamic correlates of propofol 
Pontes J C D, Medeiros C G S, Gomes O M - Efeitos do propofol na resposta contrátil do miocárdio à dopamina e dobutamina: estudo experimental em corações isolados de ratos.

Rev Bras Cir Cardiovasc 1996; 11 (4):292-8.

anesthesia in the intravenous bolus administration. Neuroscir Lett 1993; 153: 161-7.

9 Coates D P. Prys-Roberts C, Speline K R, Monk C R, Norley I - Propofol (Diprivan) by intravenous infusion with nitrous oxide: dose requirements and haemodynamic effects. Postgrag Med J 1985; 61:(Suppl.): 76-9.

10 Claeys M A, Gept E, Camu T - Haemodynamic changes during anaesthesia induced and maintained with propofol. Br J Anesth 1988; 60: 3-9.

Gauss A, Heinrich $H$, Wilder-Smith $H$ G Echocardiographic assessment of the haemodynamic effects of propofol: a comparison with etomidate and propofol. Anaesthesia 1991; 46: 99-105.

12 Wouters P F, Marcus M, Ruyter H A, Flameng W, Van Aken $\mathrm{H}$ - Cardiovascular effects of propofol in chronically instrumented dogs. Anesth Analg 1993; 76: (Suppl.): S470.

13 Puttick R M, Diedericks J, Sear J W, Glen J B, Fox P Ryder W A - Effect of graded infusion rates of propofol on regional and global left ventricular function in the dog. Br J Anaesth 1992; 69: 375-81.

Goodchild C S \& Serrao J M - Cardiovascular effects of propofol in the anasthetized dog. Br J Anaesth 1989; 63: 87-92.

15 Nascimento C, Braz J R C, Curi P R, Vane L V, Castiglia Y M M - Effects of continuous infusion of propofol on renal function in the dog: comparative study with pentobarbital sodium. Rev Bras Anestesiol 1994: 44: 163-70.

16 Medeiros C G S - Análise comparativa dos efeitos do diazepan, midazolan e propofol na contratilidade miocárdica; estudo em coraçōes isolados de ratos [Tese. Mestrado] Belo Horizonte: Curso de Pós-Graduaçāo em Cardiologia e Cirurgia Cardiovascular. Serviço do Coraçāo do Hospital São Francisco de Assis - CMC - SSVP, 1994.

17 Coetzee A, Fourie P, Coetzee J - Effect of various propofol plasma concentration on regional myocardial contractility and left ventricular afterload. Anaesth Analg 1989; 69: 473-83.

18 Riou B, Besse S, Lecarpentier $Y$, Viars $P$ - In vitro effects of propofol on rat myocardium. Anesthesiology 1992; 76: 609-16.

Di Florio T - Department of Anaesthesia, Sir Charles Gaidner Hospital. Verdun Street, Nedlands WA 6009. Australia.

20 Dingwall A E - Oculogyric crisis after day case anaesthesia. Anaesthesia, 1987; 42: 565.

21 Kebabian J W \& Calne D B - Multiple receptors for dopamine. Nature, 1979; 277: 93-6.

22

Coates D P. Monk C R, Prys-Roberts C, Turtle M Hemodynamic effects of infusions of the emulsion formulation of propofol during nitrous oxide anesthesia in humans. Anesth Analg 1987; 66: 64-70.

28 Monk C R, Coates D P. Prys-Roberts C, Turtle M J Spelina K - Haemodynamic effects of a prolonged infusion of propofol as a supplemnt to nitrous oxide anaesthesia. Br J Anaesth 1987; 59: 954-60.

29 Patrick M R, Blair I J, Feneck R O, Sebel P S - A comparison of the haemodynamic effects of propofol (Diprivan) and thiopentone in patients with coronary artery disease. Postgrad Med J1985; 61(Suppl.) 23-7.

30 Stephan H, Sonntag H, Schenk H D, Kettler D, Khambatta $\mathrm{H} \mathrm{J}$ - Effects of propofol on cardiovascular dynamics, myocardial blood flow and myocardial metabolism in patients with coronary artery disease. BrJ Anaesth 1986; 58: 969-75.

31 Vermeyen K M, Erpels F A, Janssen L A, Beeckman C P, Hanegreefs G H - Propofol-fentanyl anaesthesia for coronary bypass surgery in patients with good left ventricular function. Br J Anaesth 1987; 59: 1115-20.

32 Larsen R, Rathgeber j, Bagdahn A, Lange H, Rieke H - Effects of propofol on cardiovascular dynamics and coronary blood flow in geriatric patients: a comparasion with etomidate. Anaesthesia 1988; 43(Suppl.): 25-31. 